\title{
Projective and inductive limits of Banach spaces
}

by

\section{ED DUBINSKY (Warszawa)}

Abstract. We consider the projective and inductive limits of sequences of maps of Banach spaces. New results are given on when these are, respectively, reduced and Hausdorff. Applications are made to the question of the density of a countable intersection of dense subspaces and to the problem of constructing a Fréchet space with a given sequence of canonical maps. Also, an application to the theory of $\lambda$-nuclear spaces is indicated.

Let $T_{n}: F_{n+1} \rightarrow F_{n}, n=1,2, \ldots$ be a sequence of continuous linear maps of locally convex spaces. The projective limit of the sequence $\left(T_{n}\right)$ is the locally convex space $F=F[\tau]$ where $F$ is the vector space of all sequences $x=\left(x_{n}\right) \epsilon \prod F_{n}$ with the property that each $x_{n}=T_{n} x_{n+1}$ and $\tau$ is the induced product topology. We have the canonical projection maps $P_{n}: F \rightarrow F_{n}$ defined by $P_{n} x=x_{n}$. Obviously $P_{n}=T_{n} P_{n+1}$ for all $n$. We say that the projective limit is reduced if each $P_{n}(F)$ is dense in $F_{n}$

Let $S_{n}: E_{n} \rightarrow E_{n+1}, n=1,2, \ldots$ be a sequence of continuous linear maps of locally convex spaces. Let $Q_{n}: E_{n} \rightarrow \oplus E_{n}$ be the usual injection map which sends $x \in E_{n}$ into a sequence whose $n$th coordinate is $x$ and all other coordinates are 0 . Let $H$ be the vector subspace of $\oplus E_{n}$ generated by the elements $Q_{k} x-Q_{n-1} S_{n-1} \circ \ldots \circ S_{k} x, k<n, x \in E_{k}$. Then the $i n-$ ductive limit of the sequence $\left(S_{n}\right)$ is the topological vector space $E[\tau]$ $=\oplus E_{n} / H$. It is Hausdorff (and locally convex) if and only if $H$ is closed.

Let $T_{n}: F_{n+1} \rightarrow F_{n}, R_{n}: G_{n+1} \rightarrow G_{n}, n=1,2, \ldots$ be two sequences of continuous linear maps of Banach spaces. We say that $\left(T_{n}\right),\left(R_{n}\right)$ are equivalent if there exist isometries $U_{n}: F_{n} \rightarrow G_{n}$ such that $U_{n}^{-1} R_{n} U_{n+1}$ $=T_{n}, n=1,2, \ldots$

Let $F$ be a Fréchet space and let $\left(p_{n}\right)$ be a fundamental sequence of seminorms defining the topology. Consider the seminormed space $\left(F, p_{n}\right)$ and let $F_{p_{n}}$ be the normed space formed by taking the vector space $F / \operatorname{ker} p_{n}$ with the norm induced by $p_{n}$. Let $\hat{F}_{p_{n}}$ be the completion of $F_{p_{n}}$. There is no loss of generality if we assume that $p_{n}(x) \leqslant p_{n+1}(x)$ 
for all $n$ and $x \in F$. In this case the identity map on $F$ induces a continuous linear map $F_{p_{n+1}} \rightarrow F_{p_{n}}$ and its extension to the completions, $\hat{F}_{p_{n+1}} \rightarrow \hat{F}_{p_{n}}$ is called the $n$th canonical map of the sequence $\left(p_{n}\right)$.

If $E$ is a Banach space we shall sometimes consider more than one topology on $E$. In this case, for simplicity of notation, we adopt the convention that if no topology is mentioned then the norm topology is understood. Thus, $E^{\prime}, E^{\prime \prime}$ will stand for the first and second Banach space duals of $E$.

Liemma. Let $T_{n}: F_{n+1} \rightarrow F_{n}, n=1,2, \ldots$ be a sequence of linear continuous maps of Banach spaces with dense range. Then for each $k$, the set $\left\{b \in F_{k}: \exists\left(x_{n}\right)_{n}\right.$ with $x_{n}=T_{n} x_{n+1}$ and $\left.x_{k}=b\right\}$ is dense in $F_{k}$.

Proof. Choose $k, \varepsilon>0$ and $a \in F_{k}$. There is no loss of generality if we assume that $\left\|T_{n}\right\| \leqslant 1$ for all $n$. We construct two sequences $\left(x_{j}^{k+j-1}\right)$, $\left(x_{j}^{k+j}\right), j=1,2, \ldots$ as follows. Take $x_{1}^{k}=T_{k}\left(x_{1}^{k+1}\right)$ with $\left\|x_{1}^{k}-a\right\| \leqslant \varepsilon / 2$. Then for each $j>1$ take $x_{j}^{k+j-1}=T_{k}\left(x_{j}^{k+j}\right)$ with $\left\|x_{j}^{k+j-1}-x_{j-1}^{k+j-1}\right\| \leqslant \varepsilon / 2^{j}$. It follows that for each $n \geqslant k$, the sequence $\left(x_{j}^{n}\right)_{j}$ is Cauchy in $F_{n}$ and hence convergent to $x^{n} \in F_{n}$. By continuity we have $x^{n}=T_{n} x^{n+1}$ for all $n \geqslant k$ and $\left\|x^{k}-a\right\| \leqslant\left\|x^{k}-x_{1}^{k}\right\|+\left\|x_{1}^{k}-a\right\| \leqslant \varepsilon / 2+\varepsilon / 2=\varepsilon$. We define $x^{n}=T_{n} \circ \ldots \circ T_{k-1} x^{k}$ for $k<n$ and it follows that $\left(x^{n}\right)$ is the desired sequence.

Using this lemma, we now obtain two results; one on the density of an intersection of dense subspaces and the other characterizing reduced projective limits of sequences of Banach spaces.

Proposition 1. Let $X$ be a Banach space and $\left(X_{n}\right)_{n}$ a decreasing sequence of subspaces $\rightarrow$ for each $n \exists$ a linear continuous map $A_{n}: F_{n} \rightarrow X$ of Banach spaces with $A_{n}\left(F_{n}\right)=X_{n}$ and $A_{n}^{-1}\left(X_{n+1}\right)$ dense in $F_{n}$. Then $\cap X_{n}$ is dense in the closure of $X_{1}$.

Proof. First suppose that $A_{n}$ is not 1-1. Then we can replace it by $\bar{A}_{n}: \quad F_{n} / \operatorname{ker} A_{n} \rightarrow X$. Clearly $\bar{A}_{n}\left(F_{n} / \operatorname{ker} A_{n}\right)=A_{n}\left(F_{n}\right)=X_{n}$ and if $\Pi_{n}: F_{n} \rightarrow F_{n} /$ ker $A_{n}$ is the quotient map, then $\bar{A}_{n}^{-1}\left(X_{n+1}\right)=\Pi_{n} A_{n}^{-1}\left(X_{n+1}\right)$ which is dense because $\Pi_{n}$ is onto. Thus we may assume that $A_{n}$ is $1-1$.

Now we define $T_{n}: F_{n+1} \rightarrow F_{n}$ by $T_{n}(y)=A_{n}^{-1} A_{n+1}(y)$. This map is defined because $X_{n+1} \subset X_{n}$; it is obviously linear and its continuity follows from the closed graph theorem. Moreover, $T_{n}\left(F_{n+1}\right)$ $=A_{n}^{-1} A_{n+1}\left(F_{n+1}\right)=A_{n}^{-1}\left(X_{n+1}\right)$ so $T_{n}$ has dense range. If we define $T_{0}: F_{1} \rightarrow \bar{X}_{1}$ by $T_{0}(x)=A_{1}(x)$, then $T_{0}$ also has dense range. Applying the lemma with $k=0$ we conclude that $\bigcap_{n} T_{0} \ldots T_{n}\left(F_{n+1}\right)$ is dense in $\bar{X}_{1}$. But this intersection is clearly equal to $\bigcap_{n} A_{n+1}\left(F_{n+1}\right)=\bigcap_{n} X_{n}$.

Remark 1 . Proposition 1 is a slight generalization of an unpublished result of W. Wojtyński who assumed that $X$ was a Hilbert space, and each $A_{n}$ was a fixed map, $A: X \rightarrow X$ where $A$ was $1-1$ and had dense range. The converse of our result is clearly false for we can take each $X_{n}$ to be a fixed dense subspace which is not the image of any Banach space.

On the other hand some hypotheses are necessary for it is true that in every infinite dimensional separable Banach space there is a decreasing sequence of dense subspaces whose intersection is $\{0\}$. Indeed we need only take a dense sequence $\left(x_{n}\right)$ and perturb it slightly so that it remains dense and becomes linearly independent. Then if $X_{n}$ is the linear span of $x_{n}$, $x_{n+1}, \ldots$, the sequence $\left(X_{n}\right)$ has the desired properties.

Proposition 2. The projective limit of a sequence of linear continuous maps of Banach spaces is reduced if and only if each map has dense range.

Proof. We have the maps $T_{n}: F_{n+1} \rightarrow F_{n}$ and projective limit $F$ with canonical projections $P_{n}: F \rightarrow F_{n}, n=1,2, \ldots$

Clearly if each $P_{n}$ has dense range, then the relation $P_{n}=T_{n} P_{n+1}$ implies that $T_{n}\left(F_{n+1}\right) \supset T_{n} P_{n+1}\left(F^{\prime}\right)=P_{n}\left(F^{\prime}\right)$ so $T_{n}$ has dense range.

Conversely, if each $T_{n}$ has dense range, the lemma immediately implies that each $P_{n}$ has dense range.

Next we apply Proposition 2 to obtain a general method for constructing a Fréchet space so that it has a fundamental sequence of seminorms with preassigned canonical maps.

THEOREM. Let $T_{n}: F_{n+1} \rightarrow F_{n}, n=1,2, \ldots$ be a sequence of linear continuous maps of Banach spaces which are 1-1 and have dense range. Then $\exists$ a unique (up to isomorphism) Frechet space $F$ which has a fundamental sequence of seminorms for which the sequence of canonical maps is equivalent to $\left(T_{n}\right)_{n}$. Moreover $F$ admits a continuous norm.

Proof. Given $\left(T_{n}\right)$, let $F$ be the projective limit. A fundamental sequence of seminorms for $F$ is given by the sequence $\left(p_{n}\right)_{n}$, where

$$
p_{n}(x)=\left\|P_{n}(x)\right\|, \quad x \in F
$$

and $P_{n}: F \rightarrow F_{n}$ is the canonical map. Since $T_{n}$ is $1-1$ and $p_{n}(x)=\left\|T_{n} P_{n+1}(x)\right\|$ it follows, for a given $k$, that if $p_{k}(x)=0$, then $P_{n}(x)=0$ for all $n$ so $x=0$. Thus each $p_{n}$ is a norm. In particular $F$ admits a continuous norm. Also, $F_{p_{n}}$ is just the normed space $\left(F, p_{n}\right)$ and the canonical map $\hat{F}_{p_{n+1}} \rightarrow \hat{F}_{p_{n}}$ is the extension of the identity on $F$. Moreover, by definition, the map $P_{n}: F_{p_{n}} \rightarrow F_{n}$ is norm preserving so its extension $U_{n}: \hat{F}_{p} \rightarrow F_{n}$ is also norm preserving. By Proposition 2, $P_{n}$ has dense range so $U_{n}$ is an isometry of $\hat{F}_{p_{n}}$ onto $F_{n}$. Finally, the map $U_{n}^{-1} T_{n} U_{n+1}: \hat{F}_{p_{n+1}} \rightarrow \hat{F}_{p_{n}}$ equals the identity on $F_{p_{n+1}}$ so it is the canonical map and $F$ has the desired properties.

Since any complete locally convex space is isomorphic to the projective limit of its canonical maps, the uniqueness of $F$ follows from the 
easily checked fact that any two projective limits of two equivalent sequences of maps are isomorphic.

Remark 2. It would be interesting to know if this theorem has a converse. In [2], Proposition 1, a converse is proved for the case in which $F$ is a Fréchet space whose topology is defined by a sequence of norms which come from inner products (the assumption of nuclearity in that result is obviously irrelevant), but we are unable to prove a more general result.

Remark 3 . The theorem has an application which actually provided the motivation for these considerations. If $1 \leqslant p<q<\infty$ and $\xi \in l_{q}-l_{p}$, let $\lambda$ be the Banach space of all sequences $x=\left(x_{j}\right)$ which can be represented $x=s+\xi t, s \in l_{p}, t \in c_{0}$ (coordinatewise arithmetic) with norm inf $(\|s\|+\|t\|)$ where the inf is taken over all representations. Then $l_{p} \subset \lambda \subset l_{\alpha}$ and it can be shown that with an appropriate choice of $\xi, \exists$ a continuous linear map $T: \lambda \rightarrow \lambda$ which factors $\lambda \rightarrow l_{\infty} \stackrel{\Delta}{\rightarrow} \lambda$ with $\Delta$ diagonal, but no power of $T$ is absolutely summing. Along with the above theorem, this leads to an example of a $\lambda$-nuclear Fréchet space which is not nuclear. Details will appear subsequently in a joint paper with M. S. Ramanujan.

Finally we dualize Proposition 2 to obtain a new sufficient condition for an inductive limit to be Hausdorff. The dualization is obtained via the following well known result ([3], p. 139).

DUALITY THEOREM. If $T_{n}: F_{n+1} \rightarrow F_{n}$ is a sequence of linear continuous maps of locally convex spaces whose projective limit is reduced, then the inductive limit of the maps $T_{n}^{\prime}: \mathbb{F}_{n}^{\prime}\left[T_{k}\left(F_{n}\right)\right] \rightarrow \mathbb{F}_{n+1}^{\prime}\left[T_{k}\left(F_{n+1}\right)\right]$ is Hausdorff. (Here $T_{k}$ refers to the Mackey topology.)

PRopostition 3. Let $S_{n}: E_{n} \rightarrow E_{n+1}, n=1,2, \ldots$ be a sequence of linear continuous maps of Banach spaces ${ }^{3}$ for each $n$, the second adjoint, $S_{n}^{\prime \prime}: E_{n}^{\prime \prime} \rightarrow E_{n+1}^{\prime \prime}$ is 1-1. Then the inductive limit of $\left(S_{n}\right)$ is Hausdorff.

Proof. Let $F[\tau]$ be the projective limit of the maps of Banach spaces, $S_{n}^{\prime}: E_{n+1}^{\prime} \rightarrow E_{n}^{\prime}$ with projections $P_{n}: \mathbb{F} \rightarrow E_{n}^{\prime}$. Now $S_{n}^{\prime \prime}$ is $1-1$ if and only if $S_{n}^{\prime}\left(E_{n+1}^{\prime}\right)$ is dense in $E_{n}^{\prime}$ so by Proposition 2 each $P_{n}\left(F^{\prime}\right)$ is dense in $E_{n}^{\prime}$.

Now from the fact that $S_{n}^{\prime}$ is the adjoint of $S_{n}$ it follows that each map $S_{n}^{\prime}: E_{n+1}^{\prime}\left[\tau_{s}\left(E_{n+1}\right)\right] \rightarrow E_{n}^{\prime}\left[\tau_{s}\left(E_{n}\right)\right]$ is continuous (here $\tau_{s}$ refers to the weak topology) and hence ([3], p. 158) each of the maps $S_{n}^{\prime}: E_{n+1}^{\prime}\left[\tau_{k}\left(E_{n+1}\right)\right] \rightarrow E_{n}^{\prime}\left[\tau_{k}\left(E_{n}\right)\right]$ is continuous.

Let $F\left[\tau_{1}\right]$ be the projective limit of this last sequence of maps. Notice that the vector space $F$ and the canonical projections $P_{n}: F \rightarrow \mathbb{F}_{n}^{\prime}$ are the same as before. Since the Mackey topology $\tau_{k}\left(E_{n}\right)$ on $E_{n}^{\prime}$ is weaker than the norm topology, it follows that each $P_{n}\left(F^{\prime}\right)$ is dense in $E_{n}^{\prime}\left[\tau_{\tau_{c}}\left(E_{n}\right)\right]$ -
that is, the projective limit $F\left[\tau_{1}\right]$ is reduced.
Finally we apply the duality theorem to this last projective limit and conclude that the inductive limit of the maps $S_{n}: E_{n}\left[\tau_{k}^{\prime}\left(E_{n}^{\prime}\right)\right]$ $\rightarrow E_{n+1}\left[\tau_{k}\left(E_{n}^{\prime}\right)\right]$ is Hausdorff. Since the Mackey topology, $\tau_{k}\left(E_{n}^{\prime}\right)$, on $E_{n}$ is exactly the norm topology, we are finished.

Remark 4. The best previous theorem on this question is that of De Wilde [1] who shows that if each $S_{n}$ is 1-1 and maps the closed unit ball of $E_{n}$ onto a $\tau_{s}\left(E_{n+1}^{\prime}\right)$-compact set, then the inductive limit is Hausdorff. This hypothesis and the hypothesis of Proposition 3 do not compare in either direction as is easily seen by considering, respectively, the two maps $S: c_{0} \rightarrow c_{0}, S: l_{1} \rightarrow l_{1}$ where each map is defined by the relation,

$$
S\left(\left(x_{n}\right)\right)=\left(\frac{1}{n^{2}} x_{n}\right) .
$$

It would be interesting to have simple necessary and sufficient conditions for the inductive limit of continuous linear maps of Banach spaces to be Hausdorff - even in the ease when. each map is 1-1.

\section{References}

[1] M. De Wilde, Sur un type particular de limite inductive, Bull. Soc. Roy. Sci. Liège, $35^{\mathrm{e}}$ Année, 9-10 (1956), pp. 545-551.

[2] E. Dubinsky, A new definition of nuclear systems with applications to bases in nuclear spaces, Stud. Math. 41 (1971), pp. 149-161.

[3] H. H. Schaefer, Topological vector spaces, New York 1966.

INSTYTUT MATEMATYCZNY POLSKIEJ AKADEMII NAUK

Received April 21, 1971 